\title{
Abordagem, projeto e métodos de investigação qualitativa em contexto educacional
}

\author{
Rafael Sousa Santos ${ }^{1}$, Clara Pimenta do Vale ${ }^{2}$, Barbara Bogoni ${ }^{3}$, Poul \\ Henning Kirkegaard ${ }^{4}$ \\ ${ }^{1}$ Faculty of Architecture, University of Porto, Portugal | rfl.ss.santos@gmail.com | \\ https://orcid.org/0000-0002-6220-2631 \\ ${ }^{2}$ Faculty of Architecture, University of Porto, Portugal | clara_vale@arq.up.pt \\ https://orcid.org/0000-0003-3426-7304 \\ ${ }^{3}$ School of Architecture Urban Planning Construction Engineering, Politecnico di Milano, Italy \\ | barbara.bogoni@polimi.it | https://orcid.org/0000-0002-2597-3373 \\ ${ }^{3}$ Department of Engineering, Aarhus University, Denmark | phk@eng.au.dk| \\ https://orcid.org/0000-0003-1413-8193
}

\begin{abstract}
Resumo: Pretende-se com este artigo apresentar os resultados de uma revisão bibliográfica de tipo narrativo, tendo como foco a definição metodológica de uma investigação qualitativa em contexto educacional. Para isso, são identificados os principais elementos relativos à definição metodológica, os seus diferentes tipos e particularidades, assim como os critérios de seleção a considerar. Este artigo está organizado em três partes. Na primeira parte é considerada a abordagem de pesquisa, enquanto plano geral que conduz a investigação, referindo os tipos de abordagem existentes e as suas características específicas do ponto de vista ontológico, epistemológico e metodológico. São depois apresentados três critérios a considerar na seleção da abordagem. Por fim é referido um conjunto de particularidades das abordagens de tipo qualitativo. A segunda parte é dedicada ao projeto de pesquisa, entendido como estrutura lógica do estudo, referindo os diferentes tipos e as suas características. São depois apresentadas as estratégias de pesquisa frequentemente adotadas em estudos qualitativos, assim como os critérios de seleção. Por fim é aprofundada a estratégia de estudo de caso, tendo em conta a sua importância em estudos em contexto educacional, passando pelos aspetos que devem ser considerados na sua definição. Na terceira parte são considerados os métodos de pesquisa, referindo os diferentes procedimentos mais seguidos em estudos qualitativos, os tipos de evidência e exemplos. De seguida são assinalados três aspetos a ter em consideração na definição dos métodos de recolha e registo de dados. Por fim, é reunido um conjunto de recomendações para o processo de análise e interpretação de dados.
\end{abstract}

Palavras-chave: Investigação Qualitativa; Estratégia de Estudo de Caso; Métodos de Pesquisa; Investigação em Contexto Educacional; Definição Metodológica.

\section{Qualitative Research Approach, Design and Methods in Educational Setting}

Abstract: With this article it is intended to present the results of a narrative bibliographic review, focused on the methodological definition of a qualitative research in educational setting. For this, the main methodological elements are identified, their different types and particularities, as well as the selection criteria. This article is organized in three parts. In the first part, the research approach is considered, as a general plan that conducts the investigation, referring to the types of approach that exist and their specific characteristics from an ontological, epistemological and methodological point of view. For the selection of the type of approach, three criteria are presented. Finally, some of the particularities of qualitative approaches are pointed out. The second part is dedicated to the research design, understood as the logical structure of the study, referring to the different types and their characteristics. The research strategies frequently adopted in qualitative studies are then presented, as well as the selection criteria. Finally, taking into account its importance in studies in an educational context, the case study strategy is deepened, mentioning its particularities and the aspects to be considered in the definition process. In the third part, the research methods are addressed, referring to the different procedures most followed in qualitative studies, the types of evidence and some examples. Three important aspects are then highlighted for the definition of data collection and recording methods. Finally, a set of recommendations for the data analysis and interpretation process is presented.

Keywords: Qualitative Research; Case Study Strategy; Research Methods; Research in Educational Setting Research; Methodological Definition. 


\section{Introdução}

Este artigo apresenta os resultados de uma revisão bibliográfica de tipo narrativo, tendo como foco a definição metodológica de uma investigação qualitativa em contexto educacional, isto é, considerando os processos de ensino e aprendizagem (Hatch, 2002). Para isso, são identificados os principais elementos metodológicos - i.e. a abordagem, o projeto e os métodos de pesquisa -, os seus diferentes tipos e particularidades, assim como os critérios de seleção a considerar.

Espera-se que a descrição detalhada dos procedimentos e etapas metodológicas essenciais, elaborada a partir de um conjunto de autores de referência - como John Creswell (1994), Robert Yin (1984; 2011) ou Sharan Merriam (1998; 2009) - possa contribuir para informar e apoiar a definição metodológica de novos estudos qualitativos, em particular no contexto educacional.

Este artigo está organizado em três partes. Na primeira parte é considerada a abordagem de pesquisa, tendo em conta os seus diferentes tipos e os critérios de seleção, e termina com a enunciação de um conjunto de particularidades das abordagens qualitativas. $\mathrm{Na}$ segunda parte é abordado o projeto de pesquisa, onde se referem as diversas estratégias utilizadas em investigação qualitativa, as suas caraterísticas e critérios de seleção. É depois aprofundada a estratégia de estudo de caso, dado a sua importância na investigação em contexto educacional. A terceira e última parte é dedicada aos métodos de pesquisa, partindo dos procedimentos de recolha e registo para os de análise e interpretação.

\section{Abordagem de Pesquisa}

\subsection{Definição}

A abordagem de pesquisa ou o research approach é o termo usado para designar o plano geral que conduz a investigação. Existem três tipos de abordagens: abordagens quantitativas (QUAN), qualitativas (QUAL) e métodos mistos ou mixed methods (MM). É possível fazer uma primeira distinção entre abordagens QUAN e QUAL pelo tipo de dados que utilizam. Enquanto nas abordagens QUAN se recolhe, regista, analisa e interpreta dados mensuráveis numericamente, as abordagens QUAL são dependentes de "evidência não numérica, seja esta verbal (oral ou escrita), experiencial (filmagens ou notas), ou relacionada com artefactos (objetos, edifícios, espaços urbanos)" (Groat \& Wang, 2013, 69).

As diferenças entre abordagens QUAN e QUAL são no entanto mais substanciais. John Creswell (1994) distingue as duas a partir das suas dimensões ontológicas, epistemológicas e metodológicas, como referido na tabela 1. Ontologicamente, as abordagens QUAN assumem a realidade como sendo única e objetiva, enquanto as abordagens QUAL entendem a realidade como sendo múltipla e subjetiva. Epistemologicamente, as abordagens QUAN não consideram a interação do investigador como o objeto de estudo, ao contrário do que acontece nas abordagens QUAL. Por fim, metodologicamente, as abordagens QUAN são geralmente baseadas num método lógicodedutivo, enquanto as abordagens QUAL seguem um método lógico-indutivo.

Apesar desta dicotomia, Creswell (2014) refere que as abordagens QUAN e QUAL não devem ser entendidas como categorias rígidas, uma vez que usualmente se utilizam num mesmo estudo, seja este de que tipo for. Os estudos tendem assim a ser "mais quantitativos" ou "mais qualitativos". As abordagens MM, por sua vez, particularizam-se por associarem as características das aproximações QUAN e QUAL. Apesar de determinados autores entenderem as abordagens MM unicamente pela dimensão metódica, isto é, relativo aos métodos de recolha, registo, análise e interpretação de dados, elas são mais do que isso (Tashakkori \& Creswell, 2007a). 
Para além dos métodos combinados, são também integradas as diferentes conceções ontológicas, epistemológicas e metodológicas, de modo a tirar partido dos pontos fortes e minimizar as limitações de cada abordagem.

Tabela 1. Abordagens de pesquisa - adaptado de J. Amos Hatch $(2002,13)$.

\begin{tabular}{|c|c|c|c|}
\hline $\begin{array}{l}\text { Abordagem } \\
\text { de pesquisa }\end{array}$ & $\begin{array}{l}\text { Ontologia } \\
\text { (natureza da } \\
\text { realidade) }\end{array}$ & $\begin{array}{c}\text { Epistemologia } \\
\text { (relação do investigador } \\
\text { com o conhecimento) }\end{array}$ & $\begin{array}{l}\text { Metodologia } \\
\text { (processo de } \\
\text { obtenção do } \\
\text { conhecimento) }\end{array}$ \\
\hline Quantitativa & $\begin{array}{l}\text { a realidade é } \\
\text { objetiva e única }\end{array}$ & $\begin{array}{l}\text { o conhecimento é } \\
\text { independente do } \\
\text { investigador }\end{array}$ & métodos dedutivos \\
\hline Qualitativa & $\begin{array}{c}\text { a realidade é } \\
\text { subjetiva e múltipla }\end{array}$ & $\begin{array}{c}\text { a visão do investigador e } \\
\text { dos participantes é } \\
\text { determinante }\end{array}$ & métodos indutivos \\
\hline $\begin{array}{l}\text { Métodos } \\
\text { mistos }\end{array}$ & $\begin{array}{c}\text { a realidade é objetiva } \\
\text { ou subjetiva }\end{array}$ & $\begin{array}{l}\text { o conhecimento pode ou } \\
\text { não estar vinculado ao } \\
\text { investigador }\end{array}$ & ambos \\
\hline
\end{tabular}

\subsection{Critério}

Creswell (in Borrego, Douglas, \& Amelink, 2009) enuncia três critérios para a definição do tipo de abordagem: i) a natureza do problema de pesquisa; ii) a área de formação e a experiência do investigador; iii) o destinatário da investigação ou a audiência.

Segundo John Dewey (in Abbagnano, 1984), o problema é a declaração de uma situação que não tem um significado único ou que inclui alternativas - "em geral, qualquer possibilidade de uma alternativa" $(1984,796)$ - e que constitui o ponto de partida para qualquer indagação. Difere então da problemática, que se define como "reunião ordenada ou sistemática de problemas" (1984, 796). Creswell (2014), por sua vez, define o problema de pesquisa como uma questão ou uma preocupação a ser abordada. O problema de pesquisa pode surgir de um vazio na literatura, de conflitos entre resultados identificados na literatura, ou simplesmente de tópicos abordados de modo negligente.

Como segundo critério, Creswell (2014) refere a necessidade de ter em conta a área de formação e a experiência pessoal do investigador. Para um investigador com conhecimentos em "escrita técnico-científica, estatística, software de processamento de dados QUAN" e que recorra frequentemente a bibliografia suportada por evidência QUAN, será mais conveniente que escolha uma abordagem QUAN $(2014,51)$. No caso de investigadores que estejam acostumados a escrever em registo literário, com experiência em condução de entrevistas ou processos de observação em campo, estarão mais aptos a seguir uma abordagem QUAL. O investigador que decida utilizar uma abordagem MM, deve ter familiaridade com aspetos das abordagens QUAN e QUAL e dispor de tempo e recursos, uma vez que implicará processos complexos de recolha e análise de dados. No caso de investigações conduzidas por estudantes, Creswell (2014) recomenda a estes que considerem também as abordagens habitualmente seguidas pelos seus orientadores científicos.

A mesma questão se coloca em relação ao terceiro critério, a audiência a que se destina a investigação. Como refere Creswell $(2014,51)$, os investigadores escrevem para uma audiência específica, como "editores e leitores de revistas científicas, júris e comitês científicos de faculdades, participantes de conferências ou colegas investigadores". Nesse sentido, a tendência dessa audiência para abordagens QUAN, QUAL ou MM deve ser tida em conta. 


\subsection{Particularidades}

As abordagens QUAL são um tipo de estudo historicamente vinculado à antropologia, sociologia e às humanidades, com a particularidade de lidarem maioritariamente com formas de dados não-numéricas ou evidência empírica (Salkind, 2010). Como já foi referido, do ponto de vista ontológico as abordagens QUAL concebem a realidade como subjetiva e múltipla, isto é, a natureza do real não é independe do sujeito individual e coletivo - é gerada, de facto, através da interação social, pelo que é circunstancial e, portanto, mutável (Salkind, 2010).

Do ponto de vista epistemológico, as abordagens QUAL têm um carácter interpretativista (Hatch, 2002), na medida que cabe ao investigador interpretar o significado dos dados recolhidos. Assim, o investigador assume um papel fundamental numa abordagem QUAL, seja como observador passivo - uma vez que os principais dados são diretamente recolhidos e filtrados pelo próprio, "contaminando" o processo com os seus valores e identidade (Salkind, 2010) - seja como observador participativo - interferindo ativamente no objeto de estudo.

Outro especto das abordagens QUAL, como refere Robert Yin, é a alteração do conceito de generalidade - característico das abordagens QUAN - pela transferibilidade. O investigador, através de uma descrição detalhada e minuciosa do contexto específico, permite ao leitor fazer conexões entre o estudo e a sua própria situação. Por outro lado, atendendo ao caracter subjetivo-interpretativo deste tipo de abordagem, a documentação exaustiva dos procedimentos de recolha, registo, análise e interpretação de dados deve também ser também elaborada, com vistas à confiabilidade ou reliability da investigação.

Do ponto de vista metodológico, Creswell descreve as abordagens QUAL como sendo "multifacetadas" (por serem indutivas e dedutivas), "simultâneas" (por não seguirem uma sequencialidade linear nos processos de recolha, registo, análise e interpretação de dados) e "interativas" (pelo carácter abrangente, flexível e emergente dos procedimentos do estudo). É justamente a dimensão interativa da abordagem QUAL que grande parte dos autores sublinha como sendo uma importante particularidade deste tipo de estudo. Ao contrário da natureza usualmente fixa e pré-determinada das abordagens QUAN, a natureza das abordagens QUAL caracteriza-se pela abertura, flexibilidade e "emergência" dos seus procedimentos em função das "realidades do ambiente [em estudo]" (Hatch, 2002).

É por isso comum que as questões de pesquisa sejam refeitas no decorrer de uma investigação QUAL, que os métodos de recolha, registo, análise e interpretação de dados sejam alterados, e que até as fontes de evidência sejam substituídas, à medida que se desenvolve e aprofunda o conhecimento do problema. Como refere Hatch $(2002,10)$, o investigador QUAL "não está a juntar as peças de um puzzle do qual já conhece a figura [...] está sim a construir uma figura que vai ganhando forma à medida que as peças são recolhidas e examinadas".

\section{Projeto de pesquisa}

\subsection{Definição}

David de Vaus (2001) define o projeto ou o research design como a estrutura lógica do estudo, dentro de uma determinada abordagem de pesquisa, e que tem como principal objetivo garantir que as evidências recolhidas "permitem responder às questões de pesquisa ou testar teorias da forma menos ambígua possível" $(2001,16)$. Também no âmbito do projeto de pesquisa existem três grandes grupos, definidos pelo tipo de dados utilizados: projetos QUAN, QUAL e MM.

Como já foi referido, as investigações de caracter QUAL têm como particularidade o facto de o projeto de pesquisa poder ser emergente, isto é, de poder surgir no próprio desenvolvimento do trabalho - particularmente no âmbito educacional (Hatch, 2002). 
Como refere J. Amos Hatch (2002, 9-10), uma vez que o objetivo é entrar num "fenómeno social inserido num contexto social particular, é impossível construir um projeto a priori que tenha em conta as descobertas que surgirão do contacto entre o investigador e a realidade observada".

Ainda assim, Robert Yin (1984) defende que a investigação QUAL deve arrancar com um projeto estabelecido, ainda que provisório, que funcione como coordenada ao desenvolvimento dos trabalhos. Justamente por esta natureza de provisoriedade, e em continuidade com a proposta de Yin, Hatch (2002) recomenda que o projeto de uma investigação QUAL seja concebido com uma estrutura flexível. Deste modo, é possível ao investigador partir para a investigação com um "plano de ação" (Hatch, 2002, 38) que coordene o desenvolvimento do estudo, mas ao mesmo tempo seja sensível e permeável às transformações que as circunstâncias reais sugerirem ou ditarem.

\subsection{Estratégia}

Associado ao projeto surge a estratégia de pesquisa ou research strategy, referente à estratégia geral que ordena os procedimentos de recolha, registo, análise e interpretação de dados. No caso dos projetos QUAL, são seguidas estratégias de pesquisa narrativas, de levantamento, experimentais, arquivísticas, históricas, etnográficas ou de estudo de caso (Creswell, 2014). Para a seleção do tipo de estratégia, Yin (1984) refere três critérios: i) o tipo de questão ou problema de pesquisa; ii) o grau de controlo do investigador sobre os eventos comportamentais; iii) o grau de foco nos eventos contemporâneos e a necessidade (ou não) de considerar o contexto. A correspondência entre os critérios e os tipos de estratégia de pesquisa é ilustrada na tabela 2.

Tabela 2. Estratégias de pesquisa - adaptado de Robert Yin $(1984,5)$.

\begin{tabular}{|c|c|c|c|}
\hline $\begin{array}{l}\text { Estratégia de } \\
\text { pesquisa }\end{array}$ & $\begin{array}{l}\text { Tipo de questão } \\
\text { de pesquisa }\end{array}$ & $\begin{array}{l}\text { Requer controlo de eventos } \\
\text { comportamentais? }\end{array}$ & $\begin{array}{l}\text { Considera eventos } \\
\text { contemporâneos? }\end{array}$ \\
\hline Experimental & como, porquê & Sim & Sim \\
\hline Levantamento & $\begin{array}{c}\text { quem, o quê, } \\
\text { onde, quantos, } \\
\text { quanto }\end{array}$ & Não & Sim \\
\hline Arquivística & $\begin{array}{c}\text { quem, o quê, } \\
\text { onde, quantos, } \\
\text { quanto }\end{array}$ & Não & Sim/Não \\
\hline Histórica & como, porquê & Não & Não \\
\hline Estudo de caso & como, porquê & Não & Sim \\
\hline
\end{tabular}

\subsection{Estudo de caso}

Yin $(1984,13)$ define o estudo de caso como uma "investigação empírica que aborda um fenómeno contemporâneo no seu contexto real, especialmente quando as fronteiras entre o fenómeno e o contexto não estão claramente evidentes". Uma vantagem relevante dos estudos de caso é a possibilidade de lidar com uma grande variedade de fontes de evidência - como documentos, artefactos, entrevistas e observações - e até com diferentes tipos de dados - apesar de um grande número dos casos de estudo estarem enquadrados em abordagens QUAN, são um tipo de estratégia adequado à associação de dados QUAL e, portanto, muito usados em abordagens MM.

Nessa medida, as investigações em contexto educacional são frequentemente elaboradas a partir de uma estratégia de estudo de caso - como refere Yin (1984), mas também Sharan Merriam (1998) e Robert Stake (1995). 
Tendo em conta os critérios anteriormente definidos por Yin (1984), as estratégias de estudo de caso são indicadas quando se pretender responder questões do tipo "como" ou "porquê" e possuem um carácter exploratório. Por outro lado, a estratégias de estudo de caso são especialmente adequadas quando não é possível exercer manipulação sobre os comportamentos a analisar - ao contrário do que acontece nos experimentos, onde os comportamentos podem ser manipulados pelo investigador de forma precisa e sistemática (Yin, 1984). Por fim, as estratégias de estudos de caso são indicadas quando há relevância de considerar eventos contemporâneos e inseridos num determinado contexto.

Yin (1984) recomenda que na definição do projeto de pesquisa segundo uma estratégia de estudo de caso sejam considerados três principais aspetos: i) o número de casos a abordar; ii) a definição das unidades de análise; e iii) a ligação lógica entre os dados recolhidos e as proposições ou o problema de pesquisa. Em relação ao primeiro aspeto, quando se trata de uma investigação em contexto educacional, é usual definir cada instituição ou escola como caso de estudo autónomo. Assim, se uma investigação tiver como foco apenas uma instituição será uma estratégia de estudo de caso único ou singlecase study, se tiver várias instituições será uma estratégia de estudo de caso múltiplo ou multiple-case study.

Em relação ao segundo aspeto, a seleção das unidades de análise está vinculada à definição das próprias questões de pesquisa - se as questões não direcionam para uma determinada unidade de análise, então as questões estarão demasiado vagas ou numerosas. No entanto, mesmo quando as unidades de análise estão estabilizadas, é importante não as considerar como definitivas - pode surgir a necessidade de as rever em função de alguma descoberta durante a recolha de dados (Yin, 1984).

Em relação ao terceiro aspeto, Yin (1984) sublinha a importância de considerar como será estabelecida a ligação lógica entre os dados e as proposições ou o problema de pesquisa, isto é, qual a regra que sustenta os procedimentos de análise e interpretação. Este aspeto não só deve ser considerado como deve ser claramente explicado nos procedimentos metodológicos da investigação - esta é uma condição para a validade dos resultados do estudo.

\section{Métodos de pesquisa}

\subsection{Definição}

Depois de selecionada a abordagem e o projeto de pesquisa, resta a determinação dos métodos ou research methods mais apropriados para responder ao problema formulado, isto é, a determinação das ferramentas ou técnicas de recolha, registo, análise e interpretação de dados (Creswell, 2014). Salkind (2010) e Yin (2011) referem como principais métodos para a recolha de evidência qualitativa as entrevistas e conversas (em primeira pessoa ou em grupo), a observação (participativa ou não participativa), e a recolha de documentação e artefactos (sejam pré-existentes ou gerados como parte do processo de estudo), como referido na tabela 3.

Os métodos de análise têm, por sua vez, de ser apropriados à natureza da evidência recolhida. Em contexto qualitativo, como refere Salkind (2010) e Creswell (2014), são frequentemente utilizados métodos como análise temática (onde o investigador analisa os textos que produziu a partir de entrevistas, notas de campo e documentos, para depois aplicar códigos e desenvolver temas), análise discursiva (onde o investigador estuda a estrutura, cadência e padrões do discurso), ou análise narrativa (onde o investigador aborda o modo como as histórias de vida são construídas) (Salkind, 2010). Alguns investigadores recorrem a softwares especializados para este tipo de estudo, que se podem revelar muito úteis na organização e manuseio de grande quantidade e diversidade de evidência. 
Tabela 3. Métodos para a recolha de dados em investigação QUAN - adaptado de Robert Yin $(2011,131)$.

\begin{tabular}{|c|c|c|}
\hline $\begin{array}{l}\text { Métodos de } \\
\text { recolha de dados }\end{array}$ & Tipo de dados & Exemplos de dados \\
\hline $\begin{array}{l}\text { Entrevista e } \\
\text { conversação }\end{array}$ & $\begin{array}{l}\text { linguagem verbal e } \\
\text { corporal }\end{array}$ & $\begin{array}{l}\text { a explicação de uma pessoa sobre algum } \\
\text { comportamento ou ação; uma recordação }\end{array}$ \\
\hline Observação & $\begin{array}{l}\text { comportamentos, } \\
\text { interações sociais, ações, } \\
\text { ambiente físico }\end{array}$ & $\begin{array}{c}\text { o nível de coordenação entre duas pessoas; } \\
\text { a natureza dessa coordenação; } \\
\text { características espaciais }\end{array}$ \\
\hline Recolha & $\begin{array}{l}\text { documentos pessoais, } \\
\text { material gráfico, registos de } \\
\text { arquivo, artefactos físicos }\end{array}$ & $\begin{array}{c}\text { títulos, textos, datas e cronologias; entradas } \\
\text { num registo de arquivo }\end{array}$ \\
\hline
\end{tabular}

\subsection{Recolha e registo}

A partir de Yin (1984), Manion (1980) e Creswell $(1994,2014)$ foram sintetizados três principais aspetos a ter em consideração na definição dos métodos de recolha e registo de dados em casos de estudo QUAL: 1. a utilização de diversos métodos, fontes de evidência e, eventualmente, tipos de dados; 2 . o encadeamento explícito das evidências com o estudo; e 3. a elaboração de um banco de dados formal ou database.

Sobre o primeiro aspeto, os três autores referem a importância do caso de estudo qualitativa ser baseado em métodos múltiplos (utilizar diversos métodos para a recolha, registo, análise e interpretação de dados), de recorrer a fontes múltiplas (utilizar diversas fontes de evidência, mas convergentes em relação ao mesmo conjunto de factos) e, como defende especialmente Yin (1984), recorrer a diferentes tipos de dados (complementando dados QUAL com dados QUAN, mesmo que se trate de uma investigação QUAL). Sobre o segundo aspeto, Yin (1984) defende a importância de enunciar explicitamente as relações entre as questões de pesquisa, as fontes de evidência a utilizar, os tipos de dados a recolher e os seus propósitos. Por fim, sobre o terceiro aspeto, os três autores referem os benefícios operativos de elaborar a reunião formal das distintas evidências, sob a forma de uma base de dados - não só na fase de recolha e registo de dados, mas na fase de análise e interpretação.

\subsection{Análise e interpretação}

A análise e interpretação de dados, como refere Merriam $(2009,176)$, é o processo complexo de "dar sentido aos dados recolhidos [...] através de procedimentos de consolidação, redução e leitura". Também Hatch $(2002,148)$ define a análise e interpretação de dados como "uma procura sistemática por significado".

Merriam (1998), a partir de Bogdan e Biklen (2007), enuncia um conjunto de recomendações para o investigador durante o processo de análise e interpretação de dados em estudos QUAL. Como primeira recomendação, Merriam (1998) refere que, contrário do que uma ideia rígida de sequencialidade pode sugerir, as quatro etapas de recolha, registo análise e interpretação de dados deverão realizar-se em simultâneo, seja "dentro" ou "fora" de campo. Deste modo, o investigador tem a possibilidade de ajustar ou modificar os procedimentos de recolha e registo de dados - ou mesmo das questões de pesquisa - em função dos resultados de sessões anteriores. Para isto é necessário analisar os dados recorrentemente, ainda que de forma rudimentar.

Por outro lado, também uma parte da revisão bibliográfica deve acontecer durante e depois do trabalho de campo (Merriam, 1998). Para reforçar esta ideia, quando Creswell (2014, 246) enuncia o que considera serem os passos gerais da análise de dados, refere que "esta esquematização sugere uma abordagem linear e hierárquica construída de baixo para cima, mas eu vejo-a como interativa: as várias fases estão interrelacionadas e não se sucedem necessariamente nesta ordem". 
Merriam (1998) sugere que ideias, categorias de análise, resultados, e outros, sejam testados com os participantes, isto é, que sejam colocados à consideração dos sujeitos do estudo - sobretudo no caso dos entrevistados - elementos do estudo em desenvolvimento. Esta recomendação é especialmente pertinente em contexto educacional, tendo em conta o contributo que professores e investigadores podem trazer para 0 estudo.

Por fim, Merriam (1998) refere a importância que os dispositivos visuais (visual devices) ou as representações gráficas podem ter no processo de análise e interpretação de dados, mas também nas restantes etapas do estudo. O modo como investigador visualiza os dados recolhidos - seja por esquemas simples ou por "sofisticados modelos computacionais" (Merriam, 1998, 172) - pode influenciar ou mesmo determinar o processo e os resultados da análise e da interpretação. O recurso a estes dispositivos visuais, como refere Creswell (2014), possibilita a leitura interativa dos dados em diferentes níveis (escalas) - de quantidade, dimensão, complexidade - sobretudo no binómio geralparticular, e por isso devem ser integrados no processo de trabalho. O papel destes dispositivos é também importante na fase da comunicação dos resultados, por permitirem a síntese de informação ou dos aspetos mais relevantes do estudo.

\section{Conclusões}

Procurou-se ao longo deste artigo enunciar os principais elementos relativos à definição metodológica de uma investigação qualitativa em contexto educacional, a partir de uma revisão bibliográfica de tipo narrativo.

$\mathrm{Na}$ primeira parte é considerada a abordagem de pesquisa, enquanto plano geral que conduz a investigação, referindo os tipos de abordagem existentes e as suas características específicas do ponto de vista ontológico, epistemológico e metodológico. São depois apresentados três critérios a considerar na seleção na abordagem. Por fim é referido um conjunto de particularidades das abordagens de tipo qualitativo.

A segunda parte é dedicada ao projeto de pesquisa, entendido como estrutura lógica do estudo, referindo os diferentes tipos e as suas características. São depois apresentadas as estratégias de pesquisa frequentemente adotadas em estudos qualitativos, assim como os critérios de seleção. Por fim é aprofundada a estratégia de estudo de caso, tendo em conta a sua importância em estudos em contexto educacional, passado pelas suas particularidades aspetos que devem ser considerados na sua definição.

$\mathrm{Na}$ terceira parte são considerados os métodos de pesquisa, referindo os diferentes procedimentos mais seguidos em estudos qualitativos, os tipos de evidência e exemplos. São depois assinalados três aspetos a ter em conta na definição dos métodos de recolha e registo de dados. Por fim, é reunido um conjunto de recomendações para o processo de análise e interpretação de dados.

\section{Referências}

Bogdan, R. C., \& Biklen, S. K. (2007). Qualitative Research for Education: An Introduction to Theory and Methods. Boston: Allyn \& Bacon.

Borrego, M., Douglas, E. P., \& Amelink, C. T. (2009). Quantitative, Qualitative, and Mixed Research Methods in Engineering Education. Journal of Engineering Education, 53-66.

Creswell, J. W. (1994). Research Design: Qualitative \& Quantitative Approaches. Thousand Oaks, California: Sage Publications.

Creswell, J. W. (2014). Research Design: Qualitative, Quantitative and Mixed Methods Approaches. Thousand Oaks, California: Sage Publications.

de Vaus, D. (2001). Research Design in Social Research. London: Sage Publications.

Denzin, N. K., \& Lincoln, Y. S. (2011). Handbook of Qualitative Research. London: SAGE Publications. 
Vol. 7 | Investigação Qualitativa em Educação: Avanços e Desafios

Feilzer, M. Y. (2010). Doing Mixed Methods Research Pragmatically: Implications for the Rediscovery of Pragmatism as a Research Paradigm. Journal of Mixed Methods Research, 4, 6-16.

Goldkuhl, G. (2012). Pragmatism vs interpretivism in qualitative information systems research. European Journal of Information Systems, 21, 135-146.

Groat, L. N., \& Wang, D. (2013). Architectural Research Methods. New Jersey: John Wiley \& Sons.

Hatch, J. A. (2002). Doing qualitative research in education settings. New York: State University of New York Press.

Manion, L., Cohen, L., \& Morrison, K. (1980). Research Methods in Education. London: Routledge.

Merriam, S. B. (1998). Qualitative Research and Case Study Applications in Education. San Francisco: Jossey-Bass.

Merriam, S. B. (2009). Qualitative Research: A Guide to Design and Implementation. San Francisco: Jossey-Bass.

Salkind, N. J. (2010). Encyclopedia of Research Design. London: SAGE Publications.

Stake, R. E. (1995). The art of case study research. Thousand Oaks: Sage Publications.

Tashakkori, A., \& Creswell, J. W. (2007a). Differing Perspectives on Mixed Methods Research. Journal of Mixed Methods Research, 1, 303-308.

Tashakkori, A., \& Creswell, J. W. (2007b). Exploring the Nature of Research Questions in Mixed Methods Research. Journal of Mixed Methods Research, 1, 207-211.

Yazan, B. (2015). Three Approaches to Case Study Methods in Education: Yin, Merriam, and Stake. The Qualitative Report, 20, 134-152.

Yin, R. K. (1984). Case Study Research. London: Sage Publications.

Yin, R. K. (2011). Qualitative Research from Start to Finish. London: The Guilford Press. 